\title{
EVALUACIÓN GEOESPACIAL DEL CAMBIO DE COBERTURA Y USO DEL SUELO: CASO DEL CANTÓN LAS NAVES, PROVINCIA BOLÍVAR
}

\author{
GEOSPATIAL ASSESSMENT OF CHANGES IN LAND \\ USE/LAND COVER: THE CASE OF LAS NAVES \\ CANTON, BOLÍVAR PROVINCE
}

\begin{abstract}
Agni Lombeida ${ }^{1}$, Ma. Fernanda Calderón ${ }^{1}$, Adriana Santos ${ }^{1}$ y Cinthia Párraga ${ }^{1}$ ${ }^{1}$ Escuela Superior Politécnica del Litoral, ESPOL, Facultad de Ciencias de la Vida, Km 30.5 Vía Perimetral, Guayaquil, Ecuador

alombeid@espol.edu.ec
\end{abstract}

\section{RESUMEN}

El presente documento tiene como finalidad la evaluación del cambio de cobertura y uso del suelo dado en los últimos 12 años, aplicando los Sistemas de Información Geográfica (SIG). La metodología se estructuró en tres componentes: 1) Delimitación de la cobertura y uso del suelo del área de estudio para los años 2002, 2008 y 2014; 2) Técnicas de superposición de mapas y 3) Determinación de los cambios durante el período de análisis mediante el uso de la matriz de tabulación cruzada.

Los resultados obtenidos para los periodos de análisis 2002 -2008 y 20082014, muestran que el primer periodo presentó un cambio total del $92.3 \%$ del área de estudio, donde, el tipo de vegetación mayormente afectada fueron bosques nativos cambiando a tierras agrícolas y pastizales. Mientras, que el segundo periodo presentó un cambio total de $75.3 \%$, debido a la transformación de mosaicos agropecuarios en cultivos permanentes.

El estudio permitió conocer los cambios ocurridos en la cobertura y uso de la tierra cantón Las Naves. Asimismo, es importante evidenciar que este tipo de análisis es fundamental en los procesos de planificación y ordenamiento territorial para los gobiernos locales.

Palabras Claves: SIG, cobertura y uso del suelo, ordenamiento territorial, matriz de tabulación cruzada, superposición de mapas. 


\section{ABSTRACT}

This document aims to assess land use and land cover changes given in the past 12 years applying GIS techniques. The methodology had three components: 1 ) Determination of land use and land cover in the study area for the years 2002, 2008 and 2014; 2) Overlay maps techniques and 3) Determination of changes during the period of analysis using the cross tabulation matrix.

The results obtained for analysed periods of 2002 -2008 and 2008-2014, showed that the first one had a total change of $92.3 \%$ of the study area, where the most affected vegetation type was native forests that became agricultural lands and pasture. Meanwhile, the second period had a change of $75.3 \%$, due to the transformation of agricultural mosaics in permanent crops.

The study allowed knowing the land use and cover changes of Las Naves canton. It is also important to note that this type of analysis is essential in land use planning and management for local governments.

Keywords: GIS, land use/land cover, land-use planning, cross-tabulation, overlap maps.

\section{INTRODUCCIÓN}

El análisis de la cobertura y uso del suelo juega un papel importante en estudios para la planificación y gestión de los recursos naturales (Gong, 2012; LaFontaine et al., 2015; Pauleit \& Duhme, 2000; Zhong et al., 2012; Zhao et al., 2016), así como también, en los planes de desarrollo y ordenamiento territorial (Sylla et al., 2012; Vásquez, 1996).

A este respecto, estudios han evidenciado que una de las metodologías ampliamente utilizadas en la evaluación de los cambios de cobertura y uso del suelo es la matriz de tabulación cruzada (Santana \& Pineda, 2011; Pineda et al., 2009; Pontius et al., 2004), la cual, consiste en determinar la modificación de la vegetación natural o de los usos antropogénicos a través de un período de tiempo dentro de un área de terreno establecida (Velázquez et al., 2010). En los últimos tiempos, los avances computacionales han permitido integrar los Sistemas de Información Geográfica (SIG), para realizar un análisis más preciso de las áreas modificadas o susceptibles a cambios con el fin de comprender las causas y consecuencias de aquellos cambios (Pino, 2015; GeoCVC, 2014; Peña, 2007; Sylla et al., 2012; López, 2013; Mas \& Flamenco, 2011).

A nivel nacional, en el marco de la actualización del Plan de Desarrollo y Ordenamiento Territorial (PDyOT) los Gobiernos Autónomos Descentralizados (GAD's) llevan a cabo el análisis del cambio de cobertura y uso del suelo (GAD Municipal Las Naves, 2015; Gobierno Autónomo Parroquial de San Isidro, 2015), con el objetivo de determinar los métodos más apropiados para la utilización de cada uno de los recursos existentes en el área de estudio (Vásquez, 1996).

Por lo tanto, existe la necesidad de generar información estandarizada basada en fundamentos metodológicos de acuerdo a los requerimientos planteados en el PDyOT (SENPLADES, 2014; GAD Municipal Las Naves, 2015). En base a 
esto, la presente investigación se enfoca en la evaluación del cambio de la cobertura y uso del suelo que se ha dado en los últimos 12 años, aplicando técnicas y metodologías SIG en el cantón las Naves, provincia de Bolívar.

\section{METODOLOGÍA}

\section{1. Área de estudio}

El cantón Las Naves de la provincia de Bolívar (Figura 1) tiene una superficie de $155,90 \mathrm{Km}^{2}$, la cual, representa el 0,05\% de la superficie total del país, con una altitud que va desde 65 a 1500 m.s.n.m., una temperatura media entre 22 y $24^{\circ} \mathrm{C}$ y una precipitación media anual entre 2000 a 2500 mm (GAD Municipal Las Naves, 2015).

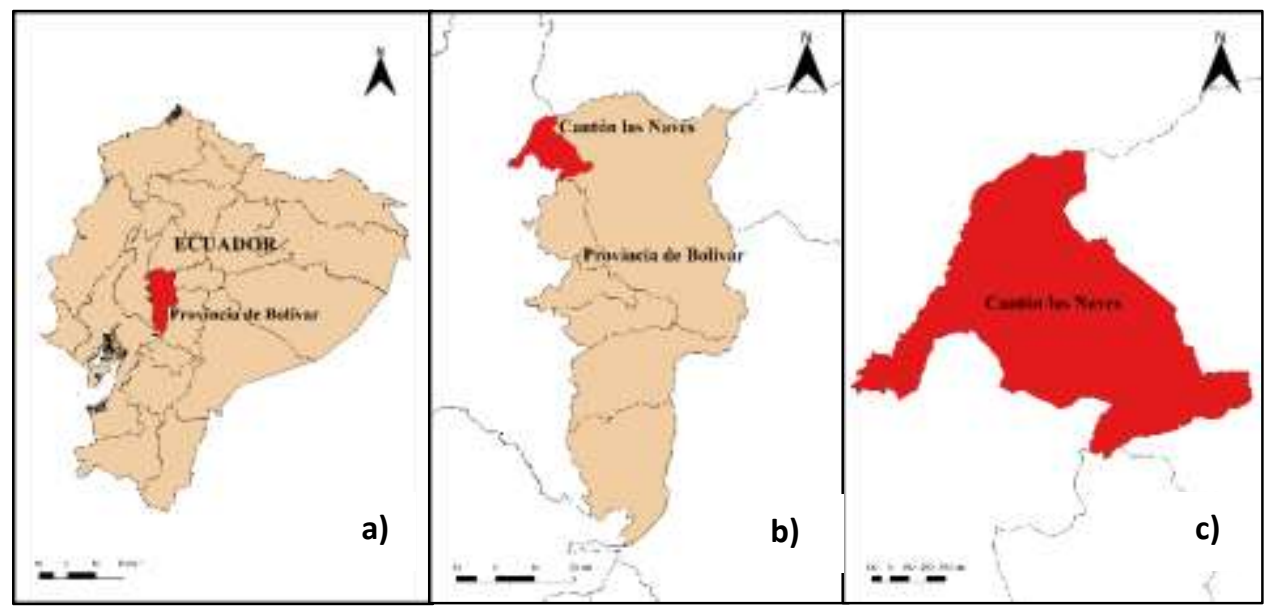

Figura 1 Ubicación del cantón Las Naves respecto al Ecuador, a) Ecuador; b) Provincia de Bolívar; c) Cantón Las Naves.

\subsection{Análisis geoespacial}

El análisis geoespacial contempló el uso de programas de acceso libre como gvSIG (gvSIG Association, 2016), QGIS (Quantum GIS Development Team, 2016) y una hoja de cálculo para la edición de los resultados. Adicionalmente, se contó con la división administrativa cantonal (INEC, 2012) y los mapas de cobertura y uso del suelo para los años 2002, 2008 y 2014 (MAGAP, 2002; SENPLADES, 2008; MAE-MAGAP, 2015), los mismos que fueron consultados en la plataforma del Sistema Nacional de Información (SNI).

La metodología de análisis se estructuró en tres componentes (Figura 2): El primer componente comprendió la delimitación de los mapas de cobertura y uso del suelo de los años 2002, 2004 y 2008 para el área de estudio, a partir de la cartografía existente a nivel nacional, puesto que tal información se encuentra en diferentes escalas fue necesario realizar una reclasificación de las categorías originales homogeneizándolas en función del uso antrópico y la cobertura natural, de acuerdo, a la clasificación adaptada para al Ecuador por (MAE-MAGAP, 2015). 


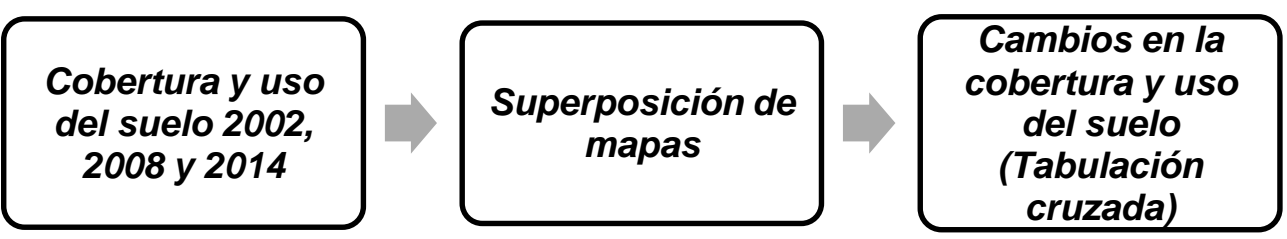

Figura 2 Diagrama de flujo de la metodología de análisis.

El segundo componente comprendió la superposición de mapas, donde se utilizó la cobertura y uso del suelo correspondiente a dos periodos 2002 y 2008 en primera instancia y en segunda instancia el 2008 y 2014, generando así, la superficie por las categorías identificadas en el paso anterior. Por último, el tercer componente comprendió la aplicación de la matriz de tabulación cruzada (Santana \& Pineda, 2011; Pineda et al., 2009; Pontius et al., 2004), entre el tiempo 1 y tiempo 2 de la cobertura y uso del suelo (Tabla 1), la cual consistió en calcular la cantidad de hectáreas transformadas de cada categoría a otras, registrando la superficie de las categorías del tiempo 1 en las filas y la superficie de las categorías del tiempo 2 en las columnas, con lo cual se obtendrán las pérdidas y ganancias (Santana \& Pineda, 2011; Pineda et al., 2009; Pontius et al., 2004).

Tabla 1 Ejemplo de la matriz de tabulación cruzada para dos tiempos con pérdidas $(P)$ y ganancias $(G)$.

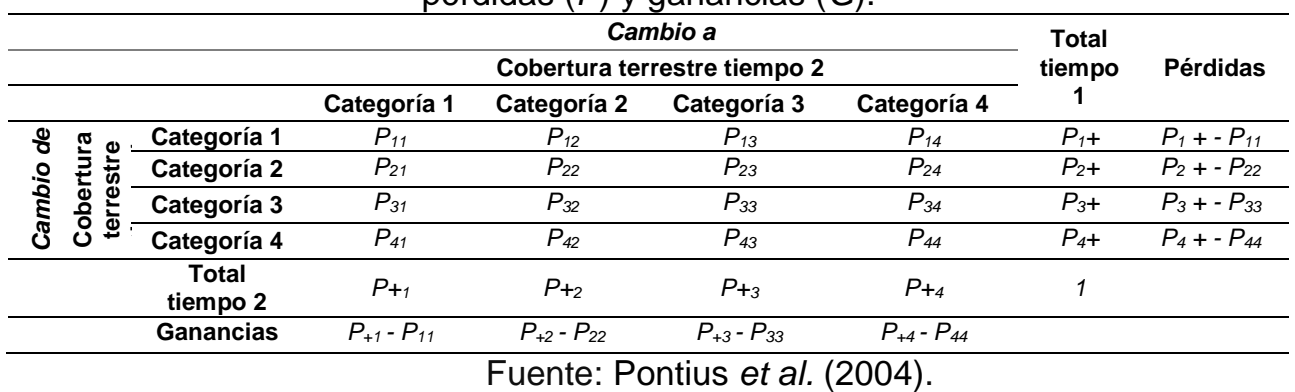

En la columna de pérdidas $(P)$ se registra la superficie de cada categoría i en las que se obtuvo pérdidas entre el tiempo 1 y tiempo 2 , siendo esta la diferencia entre la fila del total del tiempo 1 y la persistencia.

$$
P=P_{\mathrm{j}+}-P_{\mathrm{ij}},
$$

donde $\mathrm{P}$ son las pérdidas; $P_{\mathrm{j}+}$ representa la fila total del tiempo 1 y $P_{\mathrm{ij}}$ la persistencia.

En la fila de ganancias $(G)$ se registra la superficie de cada categoría j en las que se obtuvo ganancias entre el tiempo 1 y tiempo 2, significando esto la diferencia entre la columna del total del tiempo 2 y la persistencia.

$$
G=P_{+\mathrm{j}}-P_{\mathrm{ij}},
$$
persistencia.

donde $\mathrm{G}$ son las ganancias; $P_{+\mathrm{j}}$ es la columna total del tiempo 2 y $P_{\mathrm{ij}}$ es la 
La tabulación cruzada permite conocer el cambio neto $(C N)$ de cada categoría, el cual se define como el valor absoluto de la resta de las pérdidas y las ganancias para cada categoría:

$$
C N=|P-G|,
$$

donde $C N$ es el cambio neto; $P$ son las pérdidas y $G$ son las ganancias. ganancias:

El cambio total $(C T)$ de cada categoría es la suma de las pérdidas y las

$$
C T=P+G,
$$

donde $C T$ es el cambio total; $P$ son las pérdidas y $G$ son las ganancias.

El intercambio ( $I$ ) de cada categoría es la diferencia entre el cambio total $(C T)$ y el cambio neto $(C N)$.

$$
I=C N-C T,
$$

\section{RESULTADOS}

La homogenización de la clasificación de cobertura y uso del suelo resultó en ocho categorías (Figura 3): Área urbana (Au); Bosque nativo (Bn); Cuerpo de agua natural (Cag); Cultivo anual (Ca) constituido mayormente por cultivos de ciclo corto y cereales y en menor proporción por arboricultura tropical; Cultivo permanente (Cp), compuesto por árboles frutales y arboricultura tropical, y en menor proporción por cultivos de ciclo corto; Cultivo semipermanente (Cs), consta principalmente de árboles frutales y otros cultivos industriales; Mosaico agropecuario (Ma), compuesto por tierras en transición, misceláneo de frutales y misceláneo indiferenciado; y Pastizal $(\mathrm{Pa})$.

Asimismo, se observó que en la clasificación del 2002 no constan las categorías de Au y Ma, lo que resultó en un análisis de seis categorías en el 2002 y ocho categorías en el 2008 para el primer periodo. Mientras que el análisis del segundo periodo (2008-2014) se realizó para las ocho categorías descritas anteriormente, en este sentido, es importante mencionar que la información cartográfica para el segundo periodo posee mayor detalle en cuanto a la descripción de la cobertura y uso del suelo respecto a la clasificación del 2002. 


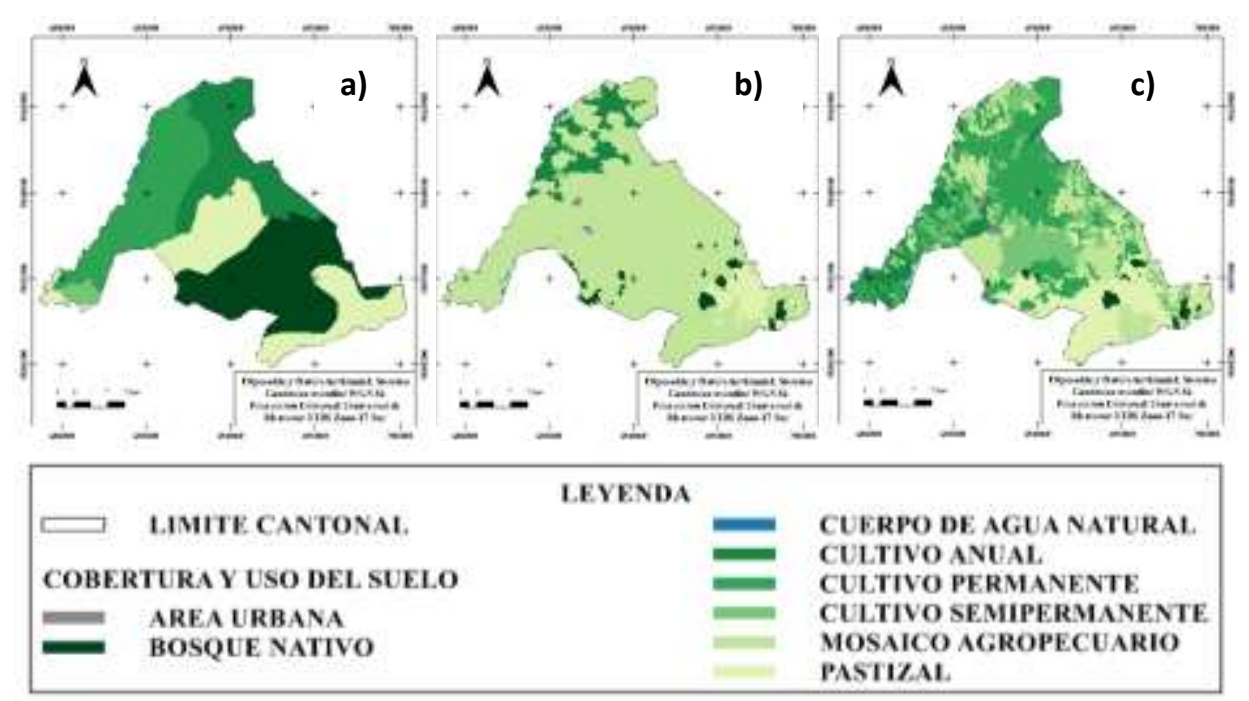

Figura 3 Mapas de cobertura y uso del suelo del cantón Las Naves de los años a) 2002; b) 2008 y c) 2014.

El primer período de análisis 2002 - 2008 (Tabla 2) mostró que el 7,65\% del área total se mantuvo persistente en el tiempo, mientras que el cambio total fue de $92,35 \%$, el mismo que se divide en un cambio neto del $83,91 \%$ y un intercambio de los procesos entre las categorías del $8,44 \%$ del territorio cantonal.

Tabla 2 Matriz de tabulación cruzada con pérdidas, ganancias y persistencias del primer periodo $(2002-2008)^{* * *}$

\begin{tabular}{|c|c|c|c|c|c|c|c|c|c|c|c|c|}
\hline & \multicolumn{10}{|c|}{ Cambio a } & \multirow{3}{*}{$\begin{array}{l}\text { Tot. } \\
2002 \\
(\%)\end{array}$} & \multirow{3}{*}{$\mathbf{P}(\%)$} \\
\hline & \multicolumn{10}{|c|}{ Cobertura terrestre 2008} & & \\
\hline & & & $\begin{array}{l}\mathrm{Au} \\
(\%)\end{array}$ & $\begin{array}{l}\text { Bn } \\
(\%)\end{array}$ & $\begin{array}{l}\text { Cag } \\
(\%)\end{array}$ & $\begin{array}{l}\mathrm{Ca} \\
(\%)\end{array}$ & $\begin{array}{l}C p \\
(\%)\end{array}$ & $\begin{array}{l}\text { Cs } \\
\text { (\%) }\end{array}$ & $\begin{array}{l}\text { Ma } \\
\text { (\%) }\end{array}$ & $\begin{array}{l}\mathrm{Pa} \\
(\%)\end{array}$ & & \\
\hline \multirow{14}{*}{$\begin{array}{l}8 \\
\text { ठ̊ } \\
\text { है } \\
\text { है }\end{array}$} & \multirow{8}{*}{ 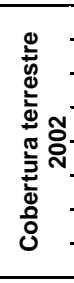 } & $\mathrm{Au}$ & - & - & - & - & - & - & - & - & - & - \\
\hline & & Bn & - & 1,63 & - & - & - & - & 26,28 & 1,52 & 29,43 & 27,80 \\
\hline & & Cag & - & - & - & 0,22 & - & - & - & - & 0,22 & 0,22 \\
\hline & & $\mathrm{Ca}$ & 0,05 & - & 0,12 & 2,67 & - & - & 16,55 & - & 19,39 & 16,72 \\
\hline & & $\mathrm{Cp}$ & 0,15 & - & 0,29 & 5,02 & 0,03 & 0,44 & 18,15 & - & 24,08 & 24,05 \\
\hline & & Cs & - & - & 0,01 & - & - & - & 1,63 & - & 1,64 & 1,64 \\
\hline & & $\mathrm{Ma}$ & - & - & - & - & - & - & - & - & - & - \\
\hline & & $\mathbf{P a}$ & 0,01 & 1,01 & 0,02 & - & - & - & 20,88 & 3,33 & 25,24 & 21,91 \\
\hline & & & & & & & & & & & 100 & 92,35 \\
\hline & & Tot. $2008(\%)$ & 0,20 & 2,64 & 0,43 & 7,90 & 0,03 & 0,44 & 83,49 & 4,86 & 100 & \\
\hline & & $\mathbf{G}(\%)$ & 0,20 & 1,01 & 0,43 & 5,24 & - & 0,44 & 83,49 & 1,52 & 92,35 & \\
\hline & & CN (\%) & 0,20 & 26,79 & 0,22 & 11,48 & 24,05 & 1,20 & 83,49 & 20,39 & 83,91 & \\
\hline & & CT (\%) & 0,20 & 28,82 & 0,65 & 21,96 & 24,05 & 2,08 & 83,49 & 23,44 & 92,35 & \\
\hline & & I (\%) & - & 2,03 & 0,43 & 10,48 & - & 0,89 & - & 3,05 & 8,44 & \\
\hline
\end{tabular}

Las pérdidas en las seis categorías encontradas en el año 2002 (Tabla 2) evidenciaron que las clases que presentaron mayores cambios fueron: $\mathrm{Bn}$ con el $27,80 \%$ el cual transformó en Ma $(26,28 \%)$ y $\mathrm{Pa}(1,52 \%)$, manteniendo una 
persistencia del $1,63 \%$. Seguido de los $\mathrm{Cp}$ con pérdidas del $24,05 \%$ que se transformó en Au $(0,15 \%)$, Cag $(0,29 \%)$, Ca $(5,02 \%)$, Cs $(0,44 \%$ en) y en $\mathrm{Ma}$ $(18,15 \%)$, con una persistencia del $0,03 \%$; y, por último, los $\mathrm{Pa}$ con pérdidas del $21,91 \%$ se transformó en Au (0,01\%), Bn (1,01\%), Cag $(0,02 \%)$ y Ma $(20,88 \%)$, y mantuvo una persistencia del 3,33\%. Así mismo, las ganancias para el año 2008 (Tabla 2) evidenciaron que el Ma con el $83,49 \%$ fue la categoría que presentó mayores ganancias, las cuales, fueron sobre el $\mathrm{Bn}(26,28 \%)$, Ca $(16,55 \%), \mathrm{Cp}$ $(18,15 \%)$, Cs $(1,63 \%)$ y $\mathrm{Pa}(20,88 \%)$.

Asimismo, las categorías que registraron pérdidas de área con respecto al cambio neto (Tabla 2) fueron: Bn con un $26,79 \%$, Pa con $20,30 \%$, Cp con $24,05 \%$, $\mathrm{Ca}$ con $11,48 \%$, Cs con $1,20 \%$. Mientras que, las categorías que registraron ganancias de área con respecto al cambio neto fueron: Ma con 83,49\%, Cag con $0,22 \%$ y Au con $0,20 \%$.

El segundo período de análisis 2008 - 2014 (Tabla 3) mostró que el 24,62\% del área total se mantuvo persistente en el tiempo, mientras que el cambio total fue de $75,38 \%$, con un cambio neto del $64,38 \%$ y un intercambio de los procesos entre las categorías con un $11 \%$ del territorio cantonal. Las pérdidas registradas en las ocho categorías en el año 2008 evidenciaron que la clase más afectada fue el Ma con pérdidas del 64,61\%, convirtiéndose en Au (0,14\%), Bn (0,13\%), Cag (0,38\%), Ca $(4,78 \%)$, Cp $(29,71 \%)$, Cs $(15,45 \%)$ y $\mathrm{Pa}(3,37 \%)$, con una persistencia del $18,93 \%$. En cuanto a las ganancias para el año 2014, la categoría que presento mayores ganancias fue $\mathrm{Cp}$ con el $33,66 \%$, ganando sobre el $\mathrm{Bn}(0,21 \%)$, Cag $(0,02 \%)$, Ca $(3,60 \%$ de), Cs $(0,12 \%$ de) y Ma $(29,71)$.

De igual manera, las categorías que registraron pérdidas de área con respecto al cambio neto fueron (Tabla 3): Ma con $60,73 \%$, Ca con $2,70 \%$ y Bn con un $0,95 \%$. Las categorías que registraron ganancias de área con respecto al cambio neto fueron: Cp con 33,66\%, Cs con 17,33\%, Pa con 13,05\%, Cag con 0,19 y Au con $0,14 \%$.

Tabla 3 Matriz de tabulación cruzada con pérdidas, ganancias y persistencias del segundo periodo $(2008-2014)^{* * *}$

\begin{tabular}{|c|c|c|c|c|c|c|c|c|c|c|c|c|}
\hline \multicolumn{11}{|c|}{ Cambio a } & \multirow{3}{*}{$\begin{array}{l}\text { Tot. } \\
2008 \\
(\%)\end{array}$} & \multirow{3}{*}{$\mathbf{P}(\%)$} \\
\hline & \multicolumn{10}{|c|}{ Cobertura terrestre 2014} & & \\
\hline & & & $\begin{array}{c}\mathrm{Au} \\
(\%)\end{array}$ & $\begin{array}{l}\mathrm{Bn} \\
(\%)\end{array}$ & $\begin{array}{l}\text { Cag } \\
(\%)\end{array}$ & $\begin{array}{c}\mathrm{Ca} \\
(\%)\end{array}$ & $\begin{array}{l}\text { Cp } \\
(\%)\end{array}$ & $\begin{array}{l}\text { Cs } \\
(\%)\end{array}$ & $\begin{array}{l}\mathrm{Ma} \\
(\%)\end{array}$ & $\begin{array}{l}\mathrm{Pa} \\
(\%)\end{array}$ & & \\
\hline \multirow{14}{*}{ 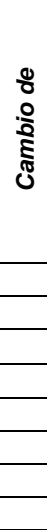 } & \multirow{14}{*}{ 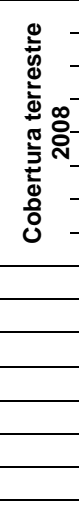 } & $\mathrm{Au}$ & 0,20 & - & - & - & - & - & - & - & 0,20 & 0,01 \\
\hline & & Bn & - & 1,56 & - & - & 0,21 & 0,05 & 0,30 & 0,51 & 2,64 & 1,08 \\
\hline & & Cag & - & - & 0,23 & - & 0,02 & 0,01 & 0,15 & - & 0,42 & 0,19 \\
\hline & & $\mathrm{Ca}$ & - & - & - & 0,31 & 3,60 & 1,46 & 2,50 & - & 7,87 & 7,56 \\
\hline & & $\mathrm{Cp}$ & - & - & - & - & 0,02 & 0,01 & - & - & 0,03 & 0,01 \\
\hline & & Cs & - & - & - & 0,08 & 0,12 & - & 0,23 & - & 0,42 & 0,42 \\
\hline & & Ma & 0,14 & 0,13 & 0,38 & 4,78 & 29,71 & 15,45 & 18,93 & 14,04 & 83,54 & 64,61 \\
\hline & & $\mathrm{Pa}$ & - & 0,01 & - & - & - & 0,78 & 0,71 & 3,37 & 4,87 & 1,50 \\
\hline & & & & & & & & & & & 100 & 75,38 \\
\hline & & Tot. 2014 (\%) & 0,34 & 1,70 & 0,61 & 5,17 & 33,69 & 17,76 & 22,81 & 17,92 & 100 & \\
\hline & & G (\%) & 0,14 & 0,14 & 0,38 & 4,86 & 33,66 & 17,76 & 3,89 & 14,55 & 75,38 & \\
\hline & & CN (\%) & 0,14 & 0,95 & 0,19 & 2,70 & 33,66 & 17,33 & 60,73 & 13,05 & 64,38 & \\
\hline & & CT (\%) & 0,15 & 1,22 & 0,57 & 12,42 & 33,67 & 18,18 & 68,50 & 16,05 & 75,38 & \\
\hline & & I (\%) & 0,01 & 0,27 & 0,38 & 9,71 & 0,01 & 0,85 & 7,77 & 3,00 & 11,00 & \\
\hline
\end{tabular}


*Área urbana (Au); Bosque nativo (Bn); Cuerpo de agua natural (Cag); Cultivo anual (Ca); Cultivo permanente (Cp); Cultivo semipermanente (Cs); Mosaico agropecuario (Ma) y Pastizal (Pa).

** Ganancia (G); Cambio neto (CN); Cambio total (CT); Intercambio (I).

\section{DISCUSIÓN}

El análisis de cambios de la cobertura y uso del suelo del primer periodo (2002-2008) evidenció que el tipo de vegetación más afectada fue el Bosque nativo, el cual se modificó principalmente en tierras agrícolas y pastizales (Santana \& Pineda, 2011). Este tipo de modificación es una actividad muy conocida en áreas rurales (ForestCarbon, 2013; González et al., 2010), dado que la frontera agrícola se ha ampliado en el territorio debido al desarrollo antropogénico (Vásquez, 1996; Galeana et al., 2009). También, es importante citar que dicha tendencia representa la principal causa de cambios de cobertura y uso del suelo a nivel mundial (González et al., 2010; Santana \& Pineda, 2011).

Asimismo, se detectó que la categoría de cultivo permanente conformada principalmente por el cultivo del café, disminuyó de manera significativa en el 2008, lo que coincide con la crisis cafetalera reportada en dicho periodo a causa del ataque de la Broca del café en las plantaciones (La Hora, 2002). Otro particular observado, fue que en el 2002 un área de cuerpo de agua natural en la zona norte del cantón cambió a cultivo anual en el 2008, se puede inferir que este fenómeno se debe a la modificación del cauce de los Ríos (Río Guabito), lo que se verifica en un cambio del tipo de cobertura como descrito por (Manson \& Jardel, 2009).

Por otro lado, el segundo periodo 2008-2014 mostró que las categorías que más ganaron en territorio fueron cultivos permanentes y pastizales y la que más perdió fue mosaico agropecuario, lo que se traduce en una disminución de cultivos anuales como maíz, transformándose a cultivos perennes como por ejemplo cacao y cítricos (González et al., 2010; Velázquez et al., 2010), las dinámicas de estos cambios permiten conocer la tendencia de producción de cultivos perennes, siendo este tipo cambio una transición común en el área agrícola como reportado por (ForestCarbon, 2013). Además, en el 2014 se evidenció el aumento de áreas con cultivos permanentes, dentro de los cuales destaca el cultivo de cacao como reportado en el informe de (Inteligencia Comercial e Inversiones, 2013), estos datos coinciden en parte con el incremento de la demanda internacional del cacao (SINAGAP, 2014), y en parte con la política de estado de impulsar el proyecto de reactivación de este cultivo desde el 2012 (MAGAP, 2012; Villon, 2014).

El análisis en general reveló, que el primer periodo presentó variaciones marcadas con porcentajes de cambio e intercambio más altos respecto al segundo período. Dichas variaciones se justifican, en primera instancia al incremento de las actividades antropogénicas (Vásquez, 1996; Galeana et al., 2009) y en segunda instancia a la calidad de información espacial de cobertura y uso del suelo, siendo en muchas partes no cercana a la realidad del territorio (Zhao et al., 2016; Yu et al., 2014).

\section{CONCLUSIONES}

Los SIG mediante la matriz de tabulación cruzada, mostró ser una herramienta idónea para evaluar los cambios de la cobertura y uso del suelo en el 
área de estudio, sea por su practicidad que por su facilidad de aplicación en diferentes zonas geográficas.

En este sentido, los resultados mostraron que el territorio ha cambiado principalmente en cuanto a los sistemas de producción, pasando de un mosaico variado de cultivos a una producción intensiva de cultivos permanentes en particular cacao y frutales. Estos cambios son una prueba tangible de la influencia de las actividades antropogénicas en los cambios de cobertura del suelo.

Por consiguiente, el presente documento constituye un insumo para futuros trabajos orientados a analizar el uso y cobertura del suelo, así como, para los tomadores de decisiones de los GAD's, en particular para el cantón las Naves y su planificación para el año 2019.

\section{REFERENCIAS}

1. ForestCarbon. (2013). Estimación de los costos de oportunidad de REDD+ (p. 2). Forest Carbon Partnership Facility.

2. GAD Municipal Las Naves. (2015). Plan de Desarrollo y Ordenamiento Territorial. Cantón Las Naves. Provincia de Bolívar. SENPLADES.

3. Galeana, J., Corona, N., \& Ordóñez, J. (2009). "Análisis dimensional de la cobertura vegetal-uso de suelo en la Cuenca del Río Magdalena". Ciencia Forestal en México, 34(105).

4. GeoCVC. (2014). Guía explicativa de la temática de cobertura y uso del suelo [Geo Portal]. Recuperado a partir de http://geocvc.co/pdf/GuiaCoberturaUso.pdf

5. Gobierno Autónomo Parroquial de San Isidro. (2015). Actualización del Plan de Desarrollo y Ordenamiento Territorial. SENPLADES.

6. Gong, P. (2012). "Remote sensing of environmental change over China: A review". Chinese Science Bulletin, 57(22), 2793-2801. https://doi.org/10.1007/s11434-012-5268-y

7. González, N., Serrano, B., Lemus, C., \& Flores, M. (2010). "Cambio de cobertura y uso del suelo en la cuenca del río Mololoa, Nayarit". Bio Ciencias, 1, 19-29.

8. gvSIG Association. (2016). gvSIG Project. Recuperado a partir de http://www.gvsig.org

9. INEC. (2012). Cantones 2011 (División Administrativa Referencial), escala $1: 50.000$.

10. Inteligencia Comercial e Inversiones. (2013). Análisis del sector cacao y elaborados. Instituto de promción de exportaciones e inversiones. Pro Ecuador. 
11. La Hora. (2002). La Broca latente en el café, cuidado con el repunte. Manta.

12. LaFontaine, J. H., Hay, L. E., Viger, R. J., Regan, R. S., \& Markstrom, S. L. (2015). "Effects of climate and land cover on hydrology in the Southeastern US: Potential impacts on watershed planning". Journal of the American Water Resources Association, 51(5), 1235-1261. https://doi.org/10.1111/1752-1688.12304

13. López, J. (2013). "Análisis del cambio de tipo de cobertura en la microcuenca urbana Hato de la Virgen ubicada en el municipio de Ibague". Revista Tumbaga, 1(8).

14. MAE-MAGAP. (2015). Protocolo metodológico para la elaboración del mapa de cobertura y uso de la tierra del Ecuador continental 2013-2014, escala 1:100.000. (p. 49). Quito.

15. MAGAP. (2002). Mapa de Cobertura y Uso de la Tierra (Caracterización de uso del suelo), escala 1:250.000.

16. MAGAP. (2012). MAGAP impulsa proyecto de reactivación del Cacao Fino y de Aroma. Recuperado a partir de http://www.agricultura.gob.ec/magapimpulsa-proyecto-de-reactivacion-del-cacao-fino-y-de-aroma/

17. Manson, R., \& Jardel, E. (2009). "Perturbaciones y desastres naturales: impactos sobre las ecorregiones, la biodiversidad y el bienestar socioeconómico". Capital natural de México, 2, 131-184.

18. Pauleit, S., \& Duhme, F. (2000). "Assessing the environmental performance of land cover types for urban planning". Landscape and Urban Planning, 52(1), 1-20.

19. Peña, J. (2007). Efectos ecológicos de los cambios de coberturas y usos del suelo en la Marina Maixa (Alicante) (Tesis Doctoral). Universidad de Alicante, Alicante, España.

20. Pineda, N., Bosque, J., Gómez, M., \& Plata, W. (2009). "Análisis de cambio del uso del suelo en el Estado de México mediante sistemas de información geográfica y técnicas de regresión multivariantes. Una aproximación a los procesos de deforestación". Investigaciones Geográficas, 69, 33-52.

21. Pino, N. (2015). Prospectiva del uso del suelo y cobertura vegetal en el Ordenamiento Territorial. Caso cantón Cuenca. (Maestria en Ordenación Territorial). Universidad de Cuenca. Facultad de Arquitectura yy Urbanismo., Cuenca, Ecuador.

22. Pontius, R. G., Shusas, E., \& McEachern, M. (2004). "Detecting important categorical land changes while accounting for persistence". Agriculture, Ecosystems and Environment, 101, 251-268. https://doi.org/10.1016/j.agee.2003.09.008 
23. Quantum GIS Development Team. (2016). Open Source Geospatial Foundation Project. Recuperado a partir de http//qgis.osgeo.org

24. Santana, G., \& Pineda, N. (2011). "Descripción de los cambios de uso y cobertura del suelo en los bosques primarios del estado de México, durante 1976-200". México: INEGI.

25. SENPLADES. (2008). Mapa de Cobertura y Uso de la Tierra (Mapa Histórico de Deforestación).

26. SENPLADES. (2014). PDyOT: instrumentos para la planificación de la inversión pública. Recuperado a partir de http://www.planificacion.gob.ec/pdyot-instrumentos-para-la-planificacionde-la-inversion-publica/

27. SINAGAP. (2014). Boletín situacional cacao (Boletín). Recuperado a partir de http://sinagap.agricultura.gob.ec/phocadownloadpap/cultivo/2014/dboletinsituacional-de-cacao-2014-actualizado.pdf

28. Sylla, L., Xiong, D., Zhang, H. Y., \& Bangoura, S. T. (2012). "A GIS technology and method to assess environmental problems from land use/cover changes: Conakry, Coyah and Dubreka region case study". The Egyptian Journal of Remote Sensing and Space Sciences, 15(1), 31-38.

29. Vásquez, A. (1996). "El Ordenamiento Territorial y los cambios en el uso de la tierra en Costa Rica". Agronomia Costarricense, 20(1), 87-94.

30. Velázquez, A., Duran, E., Larrazábal, A., López, F., \& Medina, C. (2010b). "La cobertura vegetal y los cambios de uso del suelo". México: Instituto Nacional de Ecología y Cambio Climático.

31. Villon, L. (2014). Reactivación del cacao ecuatoriano [Blog]. Recuperado a partir de http://comunidad.todocomercioexterior.com.ec/profiles/blogs/reactiviaci-ndel-cacao-ecuatoriano

32. Yu, L., Liang, L., Wang, J., Zhao, Y., Cheng, Q., Hu, L., ... Gong, P. (2014). "Meta-discoveries from a synthesis of satellite-based land-covermapping research". International Journal of Remote Sensing, 35(13), 4573-4588. https://doi.org/http://dx.doi.org/10.1080/01431161.2014.930206

33. Zhao, Y., Feng, D., Yu, L., Wang, X., Chen, Y., Bai, Y., ... Gong, P. (2016). "Detailed dynamic land cover mapping of Chile: Accuracy improvement by integrating multi-temporal data". Remote Sensing of Environment, 183, $170-185$.

34. Zhong, L., Gong, P., \& Biging, G. (2012). "Phenology-based crop classification algorithm and its implications on agricultural water use assessments in California's central valley". Photogrammetric Engineering and Remote Sensing, 78(8), 799-813. https://doi.org/10.14358/PERS.78.8.799 
